\title{
A shift in physician health
}

The Physician as Patient: A Clinical Handbook for Mental Health Professionals Michael F. Myers and Glen O. Gabbard American Psychiatric Publishing Inc.; 2008 $242 \mathrm{pp} \$ 46.00$

I t's now about 25 years since most Canadian provinces and American states developed physician health programs, many of which initially focused on physician impairment resulting from addictions to drugs and/or alcohol, with a smaller proportion related to psychiatric disorders. More recently, these programs have found their work shifting to the "disruptive physician," and to role-strain issues that arise when physicians practise in settings with clinical resources that are inadequate for delivering timely and quality care, and often have an administrative structure that is unsympathetic to the consequences to staff morale and health.

Drs. Myers and Gabbard's recent book presents an informed update of current thinking on physician health and wellness. The depth of their knowledge and clinical experience in this area is reflected in chapter themes such as "The Culture of Medicine," the discussion of the discrimination faced by minority groups among physicians, and an excellent section on the assessment and treatment of the suicidal physician.

The authors give comprehensive descriptions of the range of physician health problems. The messages are delivered with compassion and the anonymized case histories feature brief information on the physician's developmental background and the therapeutic interventions. Important recent information about evidence-based treatment of personality disorders and the benefits of a systematic approach to workplace behavioural issues are also included. Fatal Flaws, ${ }^{1}$ a recent text on personality disorders, would also be a good companion volume in understanding these disorders and approaches to treatment discussed in this book.

The well-regarded Menninger Clinic,

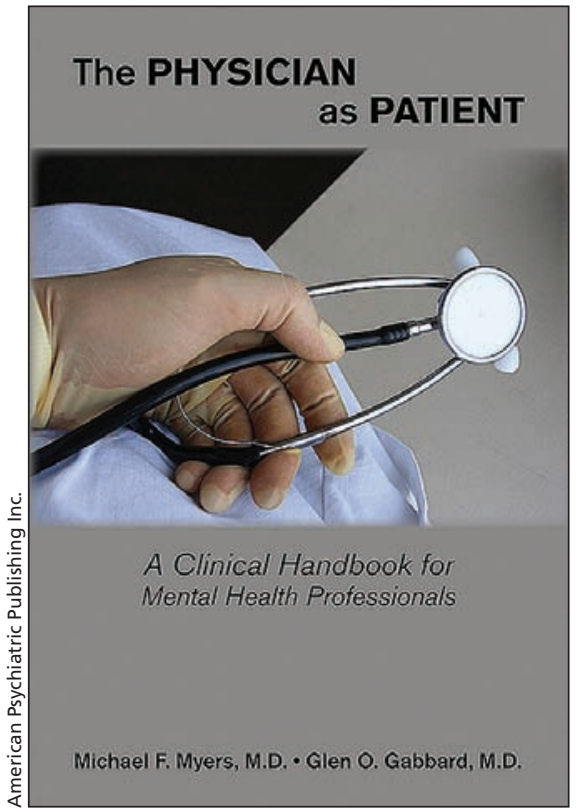

of which Dr. Gabbard is a staff member, places emphasis on the important role of mentalizing ${ }^{2}$ in any effective psychotherapy, and now uses this concept in their recently revised Professionals in Crisis program. The process of mentalizing augments conventional notions of empathy by allowing physicians to simultaneously connect more closely with the patient and selfmonitor, thus reducing risks to their own mental well-being.

When one reflects on the process of how attitudes have changed toward the treatment of physicians with addiction, it is obvious that we have a long way to go when it comes to helping colleagues with other forms of impairment. Early examples of change are appearing in some medical schools, where motivational interviewing (which matches the intervention approach with the individual's level of insight) are taught as an effective approach to chronic disease management.

Key to helping physicians in distress is the importance of reducing stigma: "There is ... a stigma that resides within the house of medicine. Judgmental, ignorant and discriminatory attitudes toward physicians living with mental illness compound their suffering, increase their isolation, foster an 'us and them' mentality, delay help-seeking, reinforce denial of illness, drive self-treatment, and heighten the risk of suicide in doctors. Changing this stigma is one of the greatest challenges for our profession in the twenty-first century. Its eradication will not only lower the number of physicians who die by suicide each year but also ensure that ill physicians get much needed treatment earlier" (p. 191).

However, one limitation of the text is the missed opportunity to discuss the advantages of incorporating more education about mental health issues into medical school curricula in order to give students the understanding and skills required to reduce this stigma, to understand and treat their patients and to be on the look out for potential problems in their colleagues and themselves (p. 206-7). In addition, the author's target audience is sometimes unclear: is it those who treat physicians or those who do regulatory assessments?

Finally, Meyers and Gabbard raise an enduring ethical challenge to those who treat physicians with serious, recurrent mental illness: the ethical conflict that situates a therapist between their patient's needs in treatment and their duty to treating them, and their duty to report impairment to regulators.

The emergence of this book can be viewed as a step away from the denial of emotional distress as a serious problem among doctors. Attitudes can change for the better, and as this book shows, these changes can benefit our patients, our colleagues and sometimes, ourselves.

\section{Ian A. Gillespie MD \\ Psychiatrist \\ Victoria, British Columbia}

\section{REFERENCES}

1. Yudofsky SC. Fatal flaws: navigating destructive relationships with people with disorders of personality and character. Arlington (VA): American Psychiatric Publishing; 2005.

2. Allen JG, Fonagy P, Bateman AW. Mentalizing in clinical practice. Arlington (VA): American Psychiatric Publishing; 2008. 